\title{
Academic Educational Data Mining predictive model for early detection of students at academic risk
}

\author{
Asst. Prof. Dr. Mohamed EL-Zeweidy \\ Higher Institute for Computers and \\ Information Systems \\ Al- Shorouk Academy \\ Cairo - Egypt \\ dr.mohamed.elzeweidy@sha.edu.eg
}

\author{
Asst. Prof. DrAhmed EL-Abbasy \\ Higher Institute for Computers and \\ Information Systems \\ Al- Shorouk Academy \\ Cairo - Egypt \\ e.mail: dr.ahmed.elabbasy@ sha.edu.eg
}

\begin{abstract}
In this research, we aimed at increasing college student retention by performing early detection of academic risk using data mining methods.

Predicting students' academic performance is critical for educational institutions because strategic programs can be planned in improving or maintaining students' performance during their period of studies in the institutions. Data mining technologies can be used to monitor students and simultaneously analyzing their academic behavior, thus providing a basis for implementing necessary intervention procedures, if required.

The paper describes and lays out a methodological framework to develop model that can be used to perform inferential queries on student performance using student academic records.

Preliminary results on Academic Educational Data Mining (AEDM) model development using Decision Tree data mining algorithms for classification are presented to classify students as early as possible, into three groups: 'low-risk' students who have a high probability of success; 'medium-risk' students who may succeed thanks to the measures taken by the university; and the 'high-risk' students who have a high probability of failing where several classification rules were generated.
\end{abstract}

\section{Key words}

Learning Analytics, Data Mining, SAS Enterprise Miner 12.3, decision tree, Educational data mining, knowledge discovery in databases (KDD). 


\section{Introduction}

Academic Educational Data Mining (AEDM) has received significant attention within higher education, holds promise for improving learning processes in formal education, and beyond as well, including being highlighted in the recently released 2011 Horizon Report [4].

This interest can, in part, be traced to the work at Purdue University which has moved the field of academic analytics from the domain of research to practical application through the implementation of Course Signals. Results from initial Course Signal pilots between fall 2007 and fall 2009 have demonstrated significant potential for improving academic achievement [1].

Despite this early success, academic analytics remains an immature field that has yet to be implemented broadly across a range of institutional types, student populations and learning technologies [2].

Two distinct research communities, Educational Data Mining (EDM) and Learning Analytics and Knowledge (LAK), have developed in response.

The first workshop on Educational Data Mining was held in 2005, in Pittsburgh, Pennsylvania. This was followed by annual workshops and, in 2008, the 1st International Conference on Educational Data Mining, held in Montreal, Quebec. Annual conferences on EDM were joined by the Journal of Educational Data Mining, which published its first issue in 2009, with Kalina Yacef as Editor. The first Handbook of Educational Data Mining was published in 2010 [7].

In the summer of 2011, the International Educational Data Mining Society (IEDMS) (http://www.educationaldatamining.org/) was formed to "promote scientific research in the interdisciplinary field of educational data mining", organizing the conferences and journal, and the free open-access publication of conference and journal articles. The EDM community brings together an inter-disciplinary community of computer scientists, learning scientists, psychometricians, and researchers from other traditions. A first review of research in EDM was presented by Romero \& Ventura [3], followed by a theoretical model proposed by Baker \& Yacef [4]. A very comprehensive review of EDM research can be found in [6]. 


\section{Related work}

With increasing competition from the private sector and reduced funding in the public sector, many higher education (HE) institutions are giving much more attention to retention and progression of students throughout their studies. Add to this the explosion in electronic data which it is now possible to collect, and the potential for Learning Analytics is clear which leads Academic Educational Data Mining (AEDM) to be used significantly within higher education.

A number of studies have been made in education data mining for discovering different pattern to improve the student's performance [8]

Kalpesh Adhatrao, Aditya Gaykar, Amiraj Dhawan, Rohit Jha and Vipul Honrao(2013) [9] developed a system which can predict the performance of students from their previous performances using concepts of data mining techniques under Classification, analyzed the data set containing information about students, such as gender, marks scored in the board examinations of classes X and XII, marks and rank in entrance examinations and results in first year of the previous batch of students. By applying the ID3 (Iterative Dichotomiser 3) and C4.5 classification algorithms on this data, predicted the general and individual performance of freshly admitted students in future examinations, for a total of 182 students, the average percentage of accuracy achieved in Bulk and Singular Evaluations is approximately 75.275 these results developed by using rapidminer.

Brijesh Kumar Baradwaj, Saurabh Pal( 2011)[13] conducted a performance analysis on 50 students whose records were taken from VBS Purvanchal University, Jaunpur (Uttar Pradesh) with the objective to study student's performance using 8 attributes. Decision tree method was used to classify the data. Study helped teachers to improve the result of the student.

Khan [12] conducted a performance study on 400 students comprising 200 boys and 200 girls selected from the senior secondary school of Aligarh Muslim University, Aligarh, India with a main objective to establish the prognostic value of different measures of cognition, personality and demographic variables for success at higher secondary level in science stream. The selection was based on cluster sampling technique in which the entire population of interest was divided into groups, or clusters, and a random sample of these clusters was selected for further analyses. It was found that girls with high socio-economic status had relatively higher academic 
achievement in science stream and boys with low socio-economic status had relatively higher academic achievement in general.

Pandey and Pal [11] conducted study on the student performance based by selecting 600 students from different colleges of Dr. R. M. L. Awadh University, Faizabad, India. By means of Bayes Classification on category, language and background qualification, it was found that whether new comer students will performer or not.

Edin Osmanbegović , Mirza Suljić (2012) [10], In this paper different techniques of data mining suitable for classification have been compared: Bayesian classifier, neural networks and decision trees. Neural networks have in many areas shown success in solving problems of prediction, approximation, function, classification and pattern recognition. Their accuracy was compared with decision trees and with the Bayesian classifier. This work is based on the survey conducted on students of the Faculty of Economics, in Tuzla, academic year 2010-2011, in which, aside from the demographic data, the data about their past success and success in college have been collected. This analysis was conducted after the training and testing of the algorithms, making it possible to draw conclusions on possible predictors of students' success ,the accuracy of NB is $76.65 \%$, MLP is $71.20 \%$ and J48 is $73.93 \%$, the results indicate that the naive bayes classifier outperforms in prediction decision tree and neural network methods, to develop this results using WEKA software, the sample of students used in this study is 257 .

\section{Methodological framework}

Academic Educational Data Mining (AEDM) considered in our work are based on supervised learning (classification) techniques given that labeled training data is available (data sets used for training purposes carry both input features describing student characteristics, as well as student academic performance).

The goal is to build an early predictive model to discriminate between students in good standing and students that are not doing well to classify students as early as possible, into three classes : 'low-risk' students who have a high probability of success; 'medium-risk' students who may succeed thanks to the measures taken by the university; and the 'high-risk' students who have a high probability of failing in all subjects in the first term of the Academic year 2013-2014 among first and second year students. 
The results of the model took place at the 8th week of the Academic Term (before the final exam by 8 weeks) thus each student has been informed by his risk level before the final exam early enough.

The Study was done on the data set of 2295 students and each student has 12 attributes. Data were analyzed using decision trees to predict student risk class depending on their academic performance (Lecture attendance- Section attendance- Midterm Score) and based on the student pre-university data (Student's Interest -High school type -High school Percentage).

Data partitioned into Two categories: the training, validation .

The training set during the learning phase allows the system to observe the type of relationships between input data and output, In this experiment the training set is $70 \%$ of the collected data set. The validation data set is used to check the degree of learning of the model in order to determine if the model is converging correctly for adequate generalization ability, in another word a validation data set with the aim of assessing their predictive accuracy and consistency with the results obtained for the training data set. This phase involves an iterative process of fitting different versions of models of training and testing data set, each time evaluating their predictive performance and then chooses the best one based on their performance, also used to evaluate the performance (accuracy) of the model. In this experiment, the validation data set is $30 \%$ of the data set. Accuracy was evaluated using the misclassification rate (MISC) and average squared error (ASE) methods for the validation data set in SAS Enterprise Miner 12.3.

Our methodological framework consists of five phases, namely Collect data, Rescale/Transform Data, Partition Data, Train Model, and Evaluate Model using Test Data. The first four phases deal with preparing the input data used to build (train) and subsequently evaluate (test) model. Trained and tested model can then be used to score incoming data.

Collect Data: data used is extracted from the student records system of the Higher Institute of Computer and information technology at El-Shorouk Academy (SHA). Identifying student information is removed during the data extraction process. Logs data of individual course events tracked by each of the tools used by an instructor in a given course shell (e.g. 12 attributes Assignments, Assessments) as well as scores (grade contributions) on gradable events recorded by the Gradebook tool. 
Rescale/Transform Data (Data Cleansing): Data is recoded / processed according to specific needs of the classification model building process. The end product is a data set of 2295 students that collects data of each course taken by each student in a given semester, augmented with (Lecture attendanceSection attendance- Midterm Score) and based on the student pre-university data (Student's Interest -High school type -High school Percentage).

The target (class) variable named Academic Risk establishes a threshold of questionable academic performance (e.g. 1 defines good academic performance "Low Risk"; 22 defines "Medium Risk"; and 3 defines poor academic performance "High Risk").

This stage is also concerned with the removal of outliers, handling of missing data, and addressing the issue of variability among courses in terms of assessment and student activity. The aforementioned variability is dealt with by replacing counts with ratios computed with respect to the average metric for the full course. An aggregated score is derived from partial (Gradebook) scores on gradable events. Once again the purpose is to shave variability across courses and compute a metric that can be used to make early predictions on student academic performance a few weeks into the semester.

Partition Data: input data is randomly divided in two datasets: a training data set, and a test data set. The training data set is used to build the model. Model is then tested using test data to compute a realistic estimate of the performance of the model on unobserved data. We use a ratio of $70 \%$ of the data used for training, and $30 \%$ testing, following standard data mining practice.

Build Predictive Model: We train our model with the training dataset, using different statistical and machine learning processes. We chose the $\mathrm{C} 4.5$ decision trees classifier as it is one of the state of the art robust classification methods that can deal with both categorical and continuous features. The C4.5 [5] decision tree algorithm is a non-parametric classifier that learns rules from data.

Model Evaluation: Trained model is evaluated using the test data to measure the predictive performance derived from the confusion matrix that yields counts of true positives (TP), true negatives (TN), false positives (FP) and false negatives (FN). Given the unbalanced nature of classes, the overall accuracy $(\mathrm{TP}+\mathrm{TN}) /(\mathrm{TP}+\mathrm{TN}+\mathrm{FP}+\mathrm{FN})$ is not a good metric for evaluating the classifier, as it is dominated by the student-in-good-standing class (TN+FP). We therefore appeal to two other accuracy metrics: sensitivity $(\mathrm{TP} /(\mathrm{TP}+\mathrm{FN}))$, which measures 
the ability of the classifier to detect the class of interest (academic risk); and specificity $(\mathrm{TN} /(\mathrm{TN}+\mathrm{FP}))$ that measures the number of false alarms raised by the classifier.

\section{Experimental setup}

A 2295 data sample corresponding to the first term of the Academic year 2013-2014 undergraduate students of the Higher Institute of Computer and information technology at El-Shorouk Academy (SHA) for the first and second year students.

Initially the size of the data set is 2295,1138 of it for the first year students and 1157 for the second year students. All the subjects for the first year students are (Introduction to computer -Discrete Math- Math1 - Technical English 1 - physics) and the subjects for the second year students are ( Math 3-data structures-Object Oriented Programming- Logic Design - Introduction To Accounting) total number of subjects are 10 subjects 5 of them for the first year students and another 5 for the second year students. Initially data is collected in an excel sheet from IT department this data is ( student code-subject name-student year-high school score-high school percentage-student status-midterm scorelecture attendance-section attendance ).In order to collect the interest of students in computer science and type of high school ,a questionnaire was prepared and distributed manually to the undergraduate students of both first and second year students. 
Academic Educational Data Mining predictive model for early detection of students at academic risk

Table 1 shows a sample of the data set used in this study.

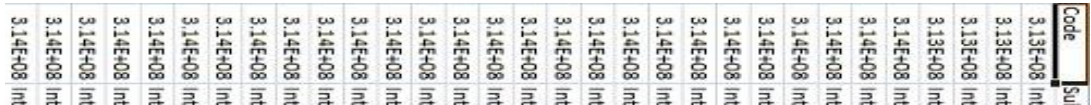

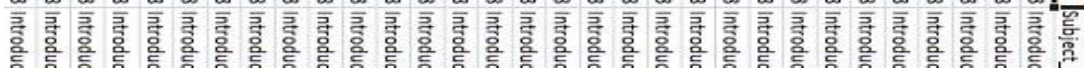

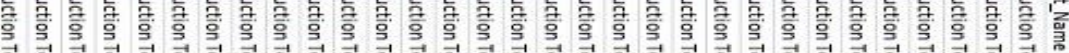

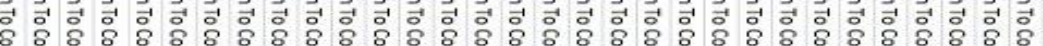

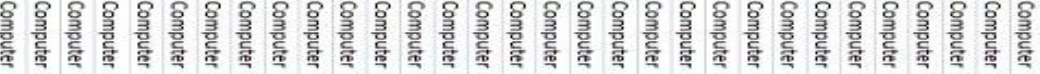

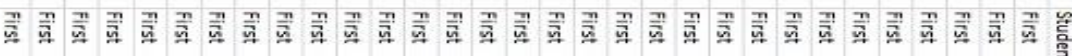

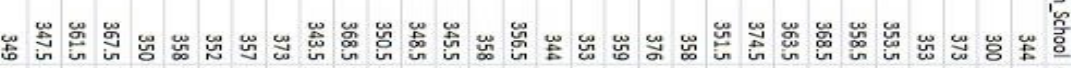

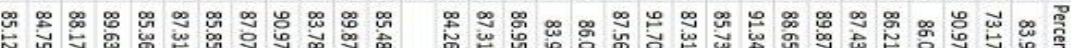

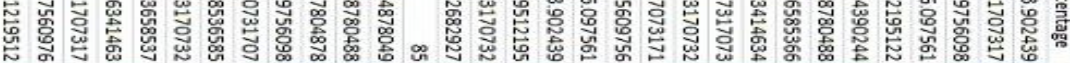

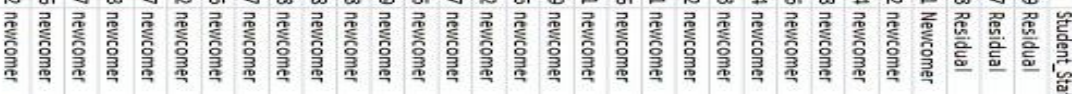
पर

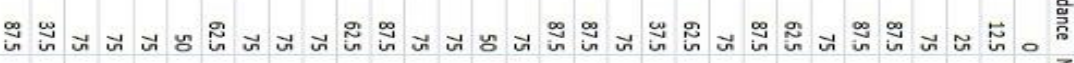

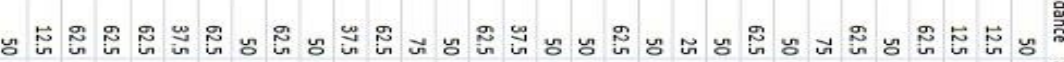

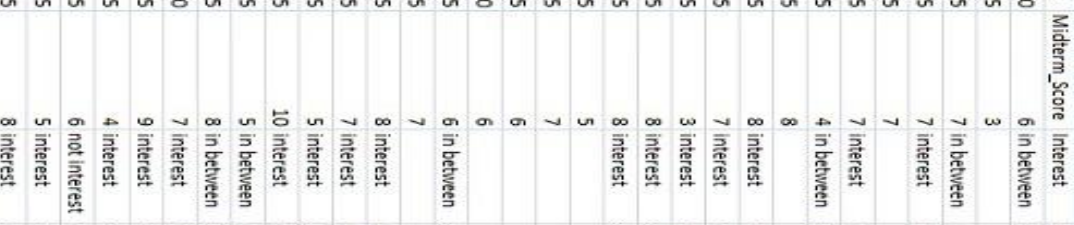

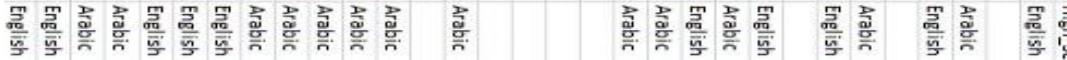

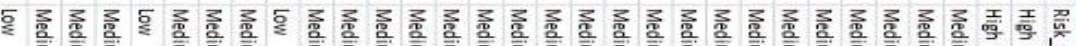

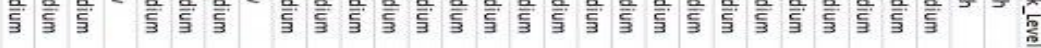

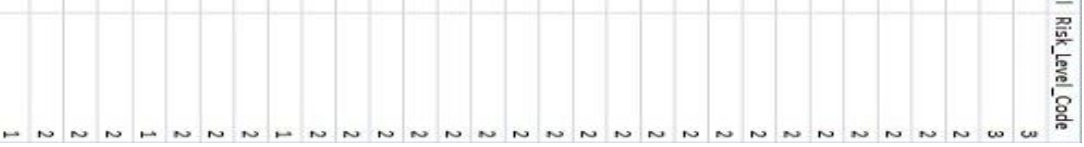


During the data selection step, only those attributes that are required for data mining were selected. The input attributes "Predictors" selected are (MIDTERM SCORE-LECTURE ATTENDANCE-SECTION ATTENDANCEHIGH SCHOOL PERCENTAGE-STUDENT INTEREST-HIGH SCHOOL TYPE-STUDENT STATUS-SUBJECT NAME). The only output attribute "Target" is (STUDENT RISK LEVEL). Table 1 shows the list of attributes with their description and possible values.

Table 2 Summarize the Predictors and Target Attribute.

\begin{tabular}{|c|c|c|}
\hline Name & Description & Possible values \\
\hline Midterm Score & $\begin{array}{l}\text { Is the score of the midterm exam of the } \\
\text { student in a particular subject }\end{array}$ & $0,1,2,3,4,5,6,7,8,9,10$ \\
\hline $\begin{array}{c}\text { Lecture } \\
\text { Attendance }\end{array}$ & $\begin{array}{r}\text { This is the Percentage of the student } \\
\text { attendance in lecture. Represent the total } \\
\text { number of days the student attended in } \\
\text { lecture for every subject }\end{array}$ & From $0 . .$. to 100 \\
\hline $\begin{array}{l}\text { Section } \\
\text { Attendance }\end{array}$ & $\begin{array}{r}\text { This is the percentage of the attendance in } \\
\text { section. Represent the total number of } \\
\text { days the student attended in Section for } \\
\text { every subject }\end{array}$ & From $0 . . .$. to 100 \\
\hline Student Status & $\begin{array}{r}\text { indicates if the student is a fresh one for } \\
\text { the year or he is a residual one } \\
\text { Ex -newcomer-Residual }\end{array}$ & $\begin{array}{ll}- & \text { New Comer } \\
\text { - } & \text { Residual }\end{array}$ \\
\hline Subject Name & $\begin{array}{l}\text { Is the name of the subject which student } \\
\text { taken in first year and second year }\end{array}$ & $\begin{array}{l}\text { Introduction to computer, } \\
\text { math3, physics, discrete } \\
\text { math, technical } \\
\text { english1, math1, data } \\
\text { structures ,Object Oriented } \\
\text { Programming, Logic Design, } \\
\text { Introduction to accounting }\end{array}$ \\
\hline $\begin{array}{l}\text { High School } \\
\text { percentage }\end{array}$ & $\begin{array}{l}\text { Is the percentage of the high school total } \\
\text { grade. }\end{array}$ & From $0 . . . .$. to 100 \\
\hline $\begin{array}{l}\text { High School } \\
\text { grade }\end{array}$ & $\begin{array}{r}\text { Is the score which student get in the high } \\
\text { school }\end{array}$ & From $0 \ldots .$. to 450 \\
\hline Risk Level & $\begin{array}{l}\text { Is the category(class)of the performance } \\
\text { of student in a particular subject. }\end{array}$ & $\begin{array}{ll}- & \text { High } \\
- & \text { Medium } \\
- & \text { Low }\end{array}$ \\
\hline
\end{tabular}

Table 2: list of all attributes with their description and possible values 


\section{Table 3. Features (predictors and target) in input dataset}

\begin{tabular}{|c|l|}
\hline Feature Type & \multicolumn{1}{|c|}{ Feature Name } \\
\hline \multirow{2}{*}{ Target } & $\begin{array}{l}\text { MIDTERM SCORE, LECTURE ATTENDANCE, SECTION } \\
\text { ATTENDANCE, HIGH SCHOOL PERCENTAGE, STUDENT } \\
\text { INTEREST, HIGH SCHOOL TYPE, STUDENT STATUS, } \\
\text { SUBJECT NAME }\end{array}$ \\
\hline & $\begin{array}{l}\text { STUDENT RISK LEVEL ACADEMIC_RISK (1 = at risk; } 0 \\
\text { student in good standing) }\end{array}$ \\
\hline
\end{tabular}

Experiments were conducted using SAS Enterprise Miner 12.3. For each of these tools a flow of execution was developed to perform the experiments. The experiments followed these guidelines:

(i) Out of the input dataset, generate five different random partitions (70\% for training, $30 \%$ for testing) by varying the random seed

(ii) Balance each training dataset by oversampling records with class ACADEMIC_RISK=1

For each balanced training dataset for the classification algorithms C4.5 Decision Tree, train a predictive model.

For the purpose of this experimental work

(iii) Using each corresponding test dataset , evaluate each classifiers' performance by measuring their predictive performance. (sensitivity, specificity)

(iv) Produce summary measures (mean and standard error) 


\section{Results and discussions}

Data was analyzed visually Using SAS Enterprise Guide and figure out the distribution of values ,Figure 2 shows the distribution of nominal attributes.

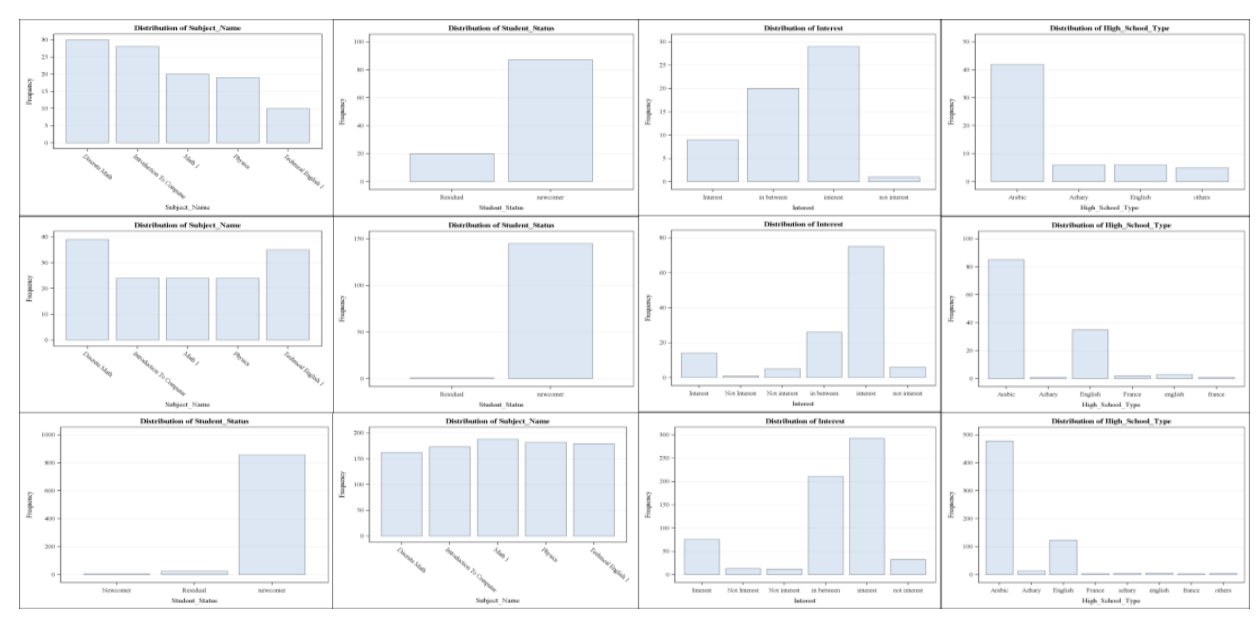

Figure 2: The distribution of nominal attributes

After analyzing the data, we feed the pruned excel sheet data set which contain all input attributes to the decision tree to rank predictors according to the strength of their relationship with depended or outcome variable (risk level).The outcome of the variable selection would be a rank list of predictors according to their importance for further analysis of the depended variable with the other methods for classification. Results of variable selection are presented in table 1.

Table 2: Variable importance

\begin{tabular}{|c|l|c|c|c|c|}
\hline Orbs & NAME & RULES\# & IMPORTANCE & VIMPORTANCE & RATIO \\
\hline 1 & $\begin{array}{l}\text { Midterm } \\
\text { Score }\end{array}$ & 2 & 1 & 1 & 1 \\
\hline 2 & $\begin{array}{l}\text { Student } \\
\text { Status }\end{array}$ & 2 & 0.68882 & 0.34642 & 0.50292 \\
\hline 3 & $\begin{array}{l}\text { Section } \\
\text { Attendance }\end{array}$ & 1 & 0.34808 & 0.16853 & 0.48416 \\
\hline
\end{tabular}

Table 2,summarize the variable name and label, the number of rules ( or splits ) in the tree that involve the variable (NRULES), the importance 
of the variable computed with the training data (IMPORTANCE), the importance of the variable computed with the validation data (VIMPORTANCE), and the ratio of VIMPORTANCE to IMPORTANCE.

The tree indicated that The most effective attributes(predictors) were: Midterm Score, student Status and Section attendance . The midterm score was the strongest attribute, then the student status ,then section attendance. The attributes such as : student interest, high school percentage, high school type, lecture attendance didn't show any clear effect for predicting student risk level, i.e. haven't any importance ( the importance of them are 0) .

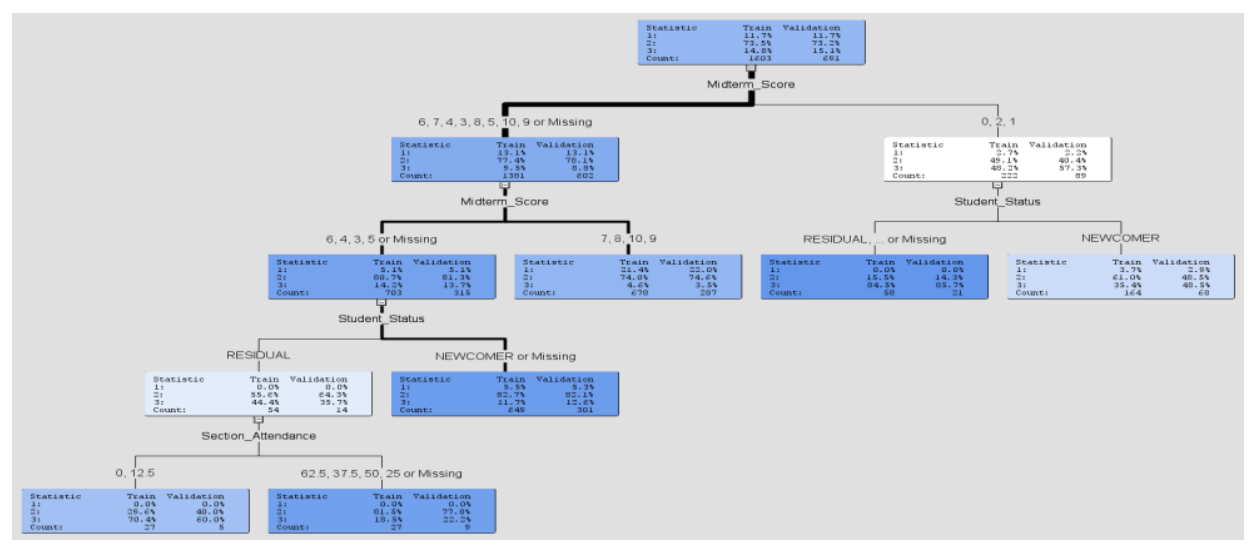

Figure 3: A decision tree diagram for predicting student risk level Figure 3 , shows the decision tree for the model , The number of levels of that tree are 4 levels and the number of leaves are 6 leaves.

The knowledge represented by decision tree can be extracted and represented in the form of IF-THEN rules. Classification tree rules can be easy to explain and used with the newly enrolled student. Rules for the decision tree for the model are given for all six terminals. 
Journal of the ACS, Vol. 9, May 2018

\section{Table 3: Classification Rules Generated By Decision Tree for the predictive model.}

\begin{tabular}{l}
\hline Node $=5$ \\
IF Midterm Score IS One OF : $7,8,10,9$ \\
THEN Tree Node Identifier $=5$, Number Of Observations $=678$, \\
Predicted: Risk Level= "MEDIUM" with probability $=0.74$. \\
\hline Node=6 \\
IF student status Is One OF $:$ RESIDUAL, FROM OUTSIDE FIRST TIME Or Missing AND \\
Midterm Score Is One Of: $0,2,1$ \\
THEN Tree Node Identifier=6, Number Of Observations=58, \\
Predicted: Risk Level=" HIGH" with probability=0.84. \\
\hline Node=7 \\
IF Student Status IS One OF: NEWCOMER AND Midterm Score Is One OF: $0,2,1$ THEN \\
Tree Node Identifier=7, Number Of Observations=164 \\
Predicted: Risk Level= "MEDIUM" with Probability=0.61 \\
\hline Node=9 \\
IF Student Status IS One Of: NEWCOMER Or Missing \\
AND Midterm Score Is One Of : $6,4,3,5$ or Missing THEN Tree Node Identifier=9, Number \\
Of Observations=649, \\
Predicted: Risk level=" MEDIUM" with probability=0.83. \\
\hline Node=14 \\
IF Student Status IS One OF: RESIDUAL AND Section Attendance Is One Of: $62.5,37.5$, \\
50,25, or missing THEN Tree Node Identifier=14, Number Of Observations=27, \\
Predicted: Risk Level="MEDIUM" with probability=0.81. \\
\hline Node=15 \\
IF Student Status Is One OF: RESIDUAL AND Section Attendance Is One Of: $0,12.5$ \\
AND Midterm Score Is One Of : $6,4,3,5$, or Missing \\
THEN Tree Node Identifier=15, Number Of Observations=27, \\
Predicted: Risk Level="HIGH" with Probability=0.70. \\
\hline
\end{tabular}

Table 3, Shows the classification rules generated by decision tree for the model, The rules for the model are given for all six terminals.

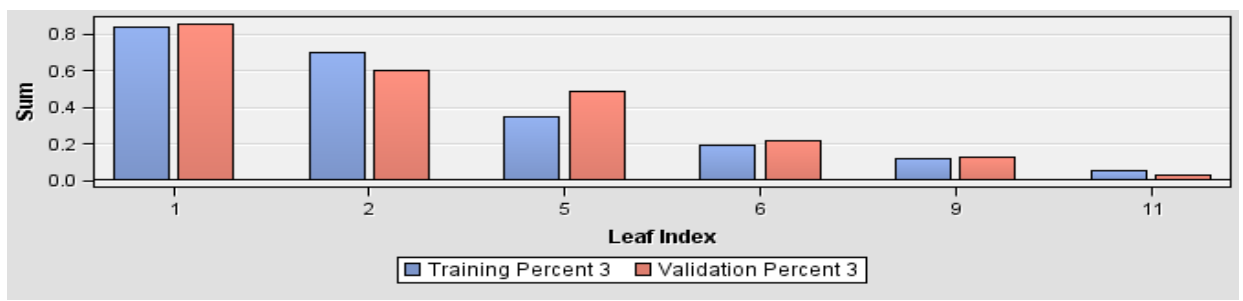

Figure 4: Leaf statistics

Figure 4 ,shows the leaf Statistics of the model, A Leaf Statistics bar chart in which the height of each bar equals the percentage of donors in 
Academic Educational Data Mining predictive model for early detection of students at academic risk

the leaf for both the training and validation data. The order of the bars is based on the percentage of donors (1's) in the training data.

\begin{tabular}{|l|l|l|l|l|l|}
\hline Node\# & Depth & $\begin{array}{l}\text { Training } \\
\text { Observations }\end{array}$ & $\begin{array}{l}\text { Training } \\
\text { Average }\end{array}$ & $\begin{array}{l}\text { Validation } \\
\text { Observations }\end{array}$ & $\begin{array}{l}\text { Validation } \\
\text { Average }\end{array}$ \\
\hline 5 & 2 & 678 & 0.05 & 287 & 0.03 \\
\hline 9 & 3 & 649 & 0.12 & 301 & 0.13 \\
\hline 7 & 2 & 164 & 0.35 & 68 & 0.49 \\
\hline 6 & 2 & 58 & 0.84 & 21 & 0.86 \\
\hline 15 & 4 & 27 & 0.7 & 5 & 0.6 \\
\hline 14 & 4 & 27 & 0.19 & 9 & 0.22 \\
\hline
\end{tabular}

\section{Table 4: Tree Leaf Report}

Table 4 , illustrating figure 4 ,i.e. Shows the tree leaf report of predictive model, These report shows that the tree has six leaves, The leaves in the table are in order from the largest number of training observations to the fewest training observations. Each node have the number of it, depth of tree on that node, number of observations of training and validation on that node, and average number of training and validation on it.

Cumulative lift is the ability of the model to learn data. Whenever the percentile of the data are increasing whenever the cumulative lift is decreasing because of the fast learning of the model to the data. Figure 5 shows the Cumulative lift for the model.

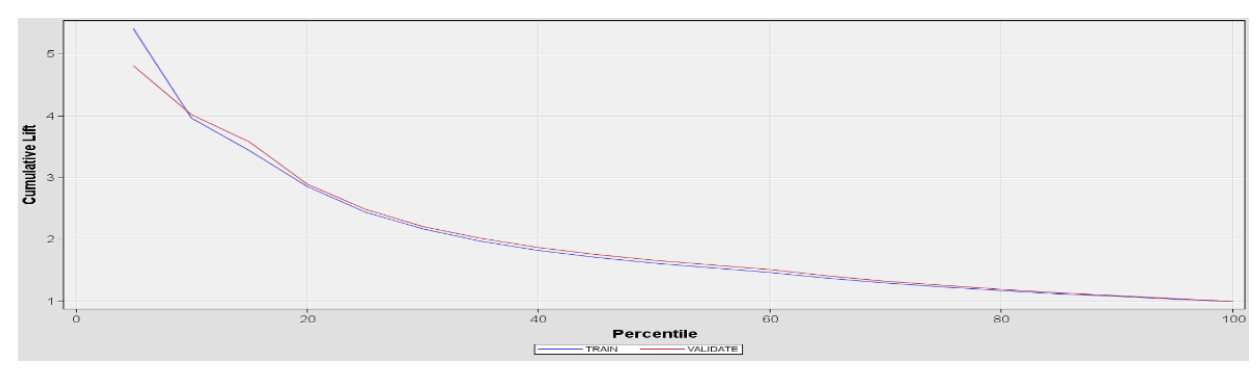

Figure 5: The Cumulative lift for the model 
Journal of the ACS, Vol. 9, May 2018

Table 5: The Assessment Score Ranking for the training set

\begin{tabular}{|c|c|c|}
\hline Percentile & $\begin{array}{c}\text { Cumulative } \\
\text { lift }\end{array}$ & $\begin{array}{c}\text { Number of } \\
\text { observations }\end{array}$ \\
\hline 5 & 5.42026 & 81 \\
\hline 10 & 3.96914 & 80 \\
\hline 15 & 3.44229 & 80 \\
\hline 20 & 2.85924 & 80 \\
\hline 25 & 2.44617 & 80 \\
\hline 30 & 2.1705 & 80 \\
\hline 35 & 1.97135 & 81 \\
\hline 40 & 1.82398 & 80 \\
\hline 45 & 1.70927 & 80 \\
\hline 50 & 1.61745 & 80 \\
\hline 55 & 1.54228 & 80 \\
\hline 60 & 1.46112 & 80 \\
\hline 65 & 1.37259 & 80 \\
\hline 70 & 1.2958 & 81 \\
\hline 75 & 1.23011 & 80 \\
\hline 80 & 1.17261 & 80 \\
\hline 85 & 1.12186 & 80 \\
\hline 90 & 1.07673 & 80 \\
\hline 95 & 1.03635 & 80 \\
\hline 100 & 1 & 80 \\
\hline
\end{tabular}

Table 5, Shows the value of cumulative lift and the number of observations on each percentile of data for the training data set.

Table 6: The Assessment Score Ranking For the validation Set

\begin{tabular}{|c|c|c|}
\hline Percentile & Cumulative lift & Number Of Observations \\
\hline 5 & 4.81567 & 35 \\
\hline 10 & 4.02004 & 35 \\
\hline 15 & 3.58573 & 34 \\
\hline 20 & 2.89406 & 35 \\
\hline 25 & 2.49014 & 34 \\
\hline 30 & 2.21227 & 35 \\
\hline 35 & 2.0193 & 34 \\
\hline 40 & 1.87014 & 35 \\
\hline 45 & 1.75739 & 34 \\
\hline
\end{tabular}


Academic Educational Data Mining predictive model for early detection of students at academic risk

\begin{tabular}{|c|c|c|}
\hline 50 & 1.66447 & 35 \\
\hline 55 & 1.58862 & 35 \\
\hline 60 & 1.51109 & 34 \\
\hline 65 & 1.41157 & 35 \\
\hline 70 & 1.32867 & 34 \\
\hline 75 & 1.25468 & 35 \\
\hline 80 & 1.19178 & 34 \\
\hline 85 & 1.13462 & 35 \\
\hline 90 & 1.08525 & 34 \\
\hline 95 & 1.03977 & 35 \\
\hline 100 & 1 & 34 \\
\hline
\end{tabular}

Table 6,Shows the values of cumulative lift and the number of observations on each percentile of data for validation data set ,Table 5 and table 6 illustration figure 5 .

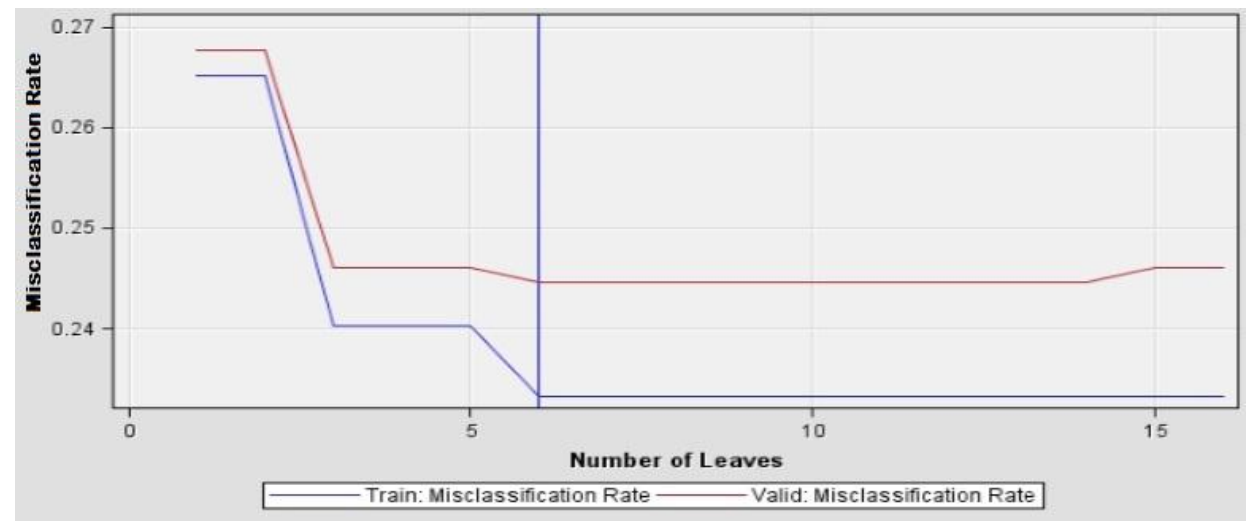

The Percentage of the correctly classified instances is often called accuracy or sample accuracy of a model.

Figure 6: The accuracy Of the model based on Misclassification rate Figure 6, Shows the accuracy of model for classification applied on data sets using Misclassification Rate, the accuracy of the model based on misclassification rate is $76 \%$.

Table 7:Misclassification Rate of leaves On training and validation sets 


\begin{tabular}{|l|l|l|}
\hline $\begin{array}{l}\text { Number Of } \\
\text { Leaves }\end{array}$ & $\begin{array}{l}\text { Misclassification } \\
\text { Rate on training }\end{array}$ & $\begin{array}{l}\text { Misclassification } \\
\text { Rate On validation }\end{array}$ \\
\hline 1 & 0.2651279 & 0.267727931 \\
\hline 2 & 0.2651279 & 0.267727931 \\
\hline 3 & 0.2401747 & 0.24602026 \\
\hline 4 & 0.2401747 & 0.24602026 \\
\hline 5 & 0.2401747 & 0.24602026 \\
\hline 6 & 0.2333125 & 0.244573082 \\
\hline 7 & 0.2333125 & 0.244573082 \\
\hline 8 & 0.2333125 & 0.244573082 \\
\hline 9 & 0.2333125 & 0.244573082 \\
\hline 10 & 0.2333125 & 0.244573082 \\
\hline 11 & 0.2333125 & 0.244573082 \\
\hline 12 & 0.2333125 & 0.244573082 \\
\hline 13 & 0.2333125 & 0.244573082 \\
\hline 14 & 0.2333125 & 0.244573082 \\
\hline 15 & 0.2333125 & 0.24602026 \\
\hline 16 & 0.2333125 & 0.24602026 \\
\hline
\end{tabular}

Table 7,illustrating Figure 6 ,i.e. shows the misclassification rate for training and validation sets on each leaf.

Figure 7: The Accuracy of the model based on Average squared Error

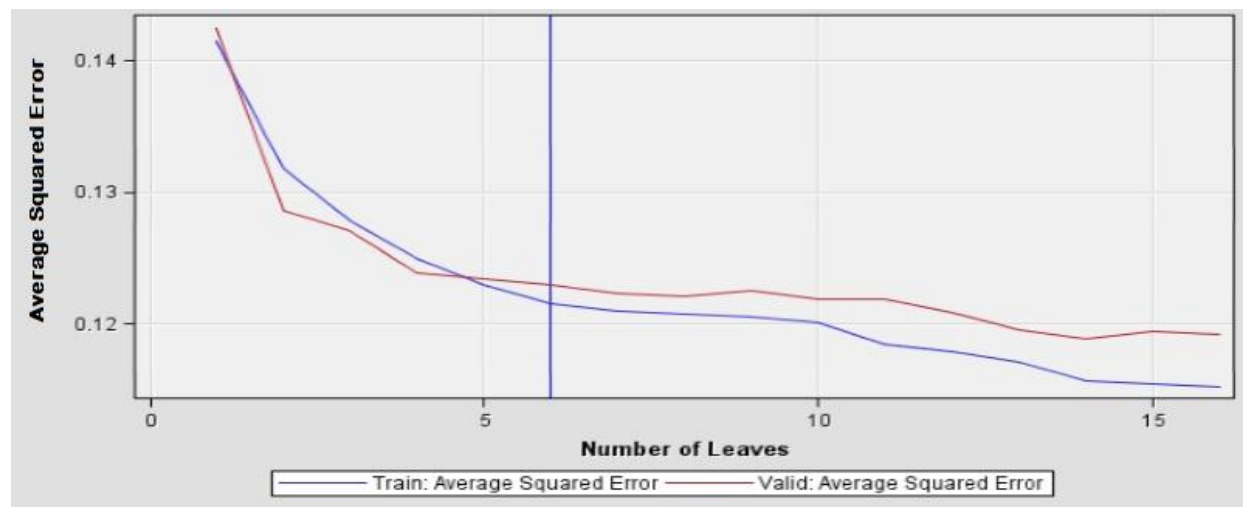

Figure 7, Shows the accuracy of a model for classification applied on data sets using Average squared Error, the accuracy of the model based on Average squared Error is $88 \%$. 
Table 8: Average Squared Error On Training and Validation Sets

\begin{tabular}{|c|c|c|}
\hline $\begin{array}{c}\text { Number Of } \\
\text { Leaves }\end{array}$ & $\begin{array}{c}\text { Average Squared Error } \\
\text { On Training Set }\end{array}$ & $\begin{array}{c}\text { Average Squared Error On } \\
\text { Validation Set }\end{array}$ \\
\hline 1 & 0.141437 & 0.142465 \\
\hline 2 & 0.131859 & 0.128631 \\
\hline 3 & 0.127857 & 0.12705 \\
\hline 4 & 0.124975 & 0.123922 \\
\hline 5 & 0.123066 & 0.123468 \\
\hline 6 & 0.121557 & 0.122986 \\
\hline 7 & 0.121041 & 0.122396 \\
\hline 8 & 0.120828 & 0.122133 \\
\hline 9 & 0.12056 & 0.122546 \\
\hline 10 & 0.120091 & 0.121929 \\
\hline 11 & 0.118468 & 0.121936 \\
\hline 12 & 0.117973 & 0.120966 \\
\hline 13 & 0.117222 & 0.119627 \\
\hline 14 & 0.115731 & 0.118974 \\
\hline 15 & 0.115468 & 0.11948 \\
\hline 16 & 0.115351 & 0.11929 \\
\hline
\end{tabular}

Table 8,illustrating Figure 7 ,i.e. shows Average Squared Error for training and validation sets on each leaf.

Table 9: Fit Statistics of a model

\begin{tabular}{|r|r|r|r|}
\hline Fit Statistics & Statistics Label & Train & Validation \\
\hline _MISC_ & Misclassification Rate & 0.23 & 0.24 \\
\hline _ASE_ & Average Squared Error & 0.12 & 0.12 \\
\hline
\end{tabular}

Table 9, Shows the fit statistics (Misclassification rate and average squared error) for the predictive model on training and validation sets.

To compute the accuracy of a model based on misclassification rate and average squared error , the accuracy $=1$ - misclassification rate , and $=1$ average squared error. 
Table 10: Accuracy Of a Model on validation and training sets based on misclassification

\begin{tabular}{|c|c|c|}
\hline Data Set & $\begin{array}{c}\text { Accuracy based on } \\
\text { misclassification rate }\end{array}$ & $\begin{array}{c}\text { Accuracy based on } \\
\text { average squared } \\
\text { error }\end{array}$ \\
\hline Validation Set & $76 \%$ & $88 \%$ \\
\hline Training Set & $77 \%$ & $88 \%$ \\
\hline
\end{tabular}

Table 10, Shows the accuracy percentage of a model based on misclassification rate and average squared error on a validation and training sets.

Table 11: All results of a model on a validation set.

\begin{tabular}{|c|c|c|c|c|c|c|}
\hline $\begin{array}{c}\text { Model } \\
\text { Name }\end{array}$ & MISC & ASE & $\begin{array}{c}\text { MISC } \\
\text { Accuracy }\end{array}$ & $\begin{array}{c}\text { ASE } \\
\text { Accuracy }\end{array}$ & $\begin{array}{c}\text { Tree } \\
\text { leaves \# }\end{array}$ & $\begin{array}{c}\text { Tree } \\
\text { levels \# }\end{array}$ \\
\hline Model & 0.24 & 0.12 & $76 \%$ & $88 \%$ & 6 & 4 \\
\hline
\end{tabular}

Table 11: Summarize all results of a model on a validation set, Number of tree levels of the model are 4 levels, Number of tree leaves of a model are 6 leaves, MISC(Misclassification rate) is 0.24, $\mathrm{ASE}$ (Average Squared Error) is 0.12 ,Accuracy of a model based on MISC $=1-\mathrm{MISC}=76 \%$, And accuracy of a model based on ASE $=1-\mathrm{ASE}=88 \%$.

\section{Conclusion}

This paper reports on the goals and objectives of the Academic Analytic, providing a detailed description of the methodology used to develop predictive model in academic analytics using SAS Enterprise Miner 12.3. to early detect potential weak "at-risk" students, so that the instructors can take an appropriate action towards them. For instance, they can give advice to prevent failure in the examination or early desertion of studies. In this work, we used decision tree technique to predict the student's risk level in all subjects for first and second year students. The model was tested on a real case study of computer science department from ElShorouk Academy, the results of this study shows that from the validation 
data set the accuracy of the model based on MISC is $76 \%$, and the accuracy of it based on ASE is $88 \%$. On working on performance, Many attributes have been tested and some of them are found effective on the performance prediction. The midterm score was the strongest attribute, then the student status and then the section attendance. But another attributes didn't show any clear effect on outcome variable (risk level).

Future work can be conducted to build a user-friendly software of instructors using the concept of decision tree method in which an instructor will just have to enter the details of the students and it will display the possible result (risk level) of those students in final exams and then make alert on risky students.

Also a flexible system can be made in which we can add the data set dynamically and the system updates its results automatically, another future work the same concept can be extended for student internal selection within the faculty for the different departments, future work can be performed for improving the classification accuracies by the usage of different data mining techniques such as (ANN) with more distinctive attributes

\section{Acknowledgements}

This research is supported by EL Shourouk Academy.. 


\section{References}

[1] Arnold, Kimberly E. "Signals: Applying Academic Analytics", EDUCAUSE Quarterly, vol.33, no. 1, 2010.

[2] Baepler, P., Murdoch, C.J. (2010, July). Academic Analytics and Data Mining in Higher Education. International Journal for the Scholarship of Teaching and Learning vol. 4, no. 2, pp. 1-9 (July 2010) ISSN 1931-4744 @ Georgia Southern University.

[3] George Siemens, Ryan S J.d. Baker,'Learning Analytics and Educational Data Mining: Towards Communication and Collaboration", ACM 1-58113-000-0/00/0010, Conference'10, Month 1-2, 2010

[4] Johnson, L., Smith, R., Willis, H., Levine, A., and Haywood, K., (2011). The 2011 Horizon Report.Austin, Texas: The New Media Consortium.

[5]. Baker, R.S.J.d., Yacef, K. (2009) The State of Educational

Data Mining in 2009: A Review and Future Visions. Journal of Educational Data Mining, 1 (1), 3-17.

[6]. Romero, C., Ventura, S. (2010) Educational Data Mining: A Review of the State-of-the-Art. IEEE Transaction on Systems, Man, and Cybernetics, Part $\mathrm{C}$ : Applications and

Reviews. 40 (6), 601-618.

[7]. Romero, C., Ventura, S., Pechenizky, M., Baker, R. (2010) Handbook of Educational Data Mining. 2010. Editorial Chapman and Hall/CRC Press, Taylor \& Francis Group. Data Mining and Knowledge Discovery Series.

[5] Quinlan, J.R., C4.5 : programs for machine learning. The Morgan Kaufmann series in machine learning. 1993, San Mateo, Calif.: Morgan Kaufmann Publishers.

[6] U.S. Department of Education, National Center for Education Statistics. Integrated Postsecondary Education Data System, Fall 2010. Retrieved February 15, 2011 from http://nces.ed.gov/collegenavigator.

[7] Vapnik, V.N., The nature of statistical learning theory. $2^{\text {nd }}$ ed. Statistics for engineering and information science. 2000, New York: Springer. 
Academic Educational Data Mining predictive model for early detection of students at academic risk

[8] Hall, M., Frank, E., Holmes, G.,Pfahringer, B., Reutemann, P., Witten, I.H. (2009); The WEKA Data Mining Software: An Update; SIGKDD Explorations, 11(1)

[9] Kalpesh Adhatrao, Aditya Gaykar, Amiraj Dhawan, Rohit Jha and Vipul Honrao . PREDICTING STUDENTS' PERFORMANCE USING ID3 AND C4.5 CLASSIFICATION ALGORITHMS. International Journal of Data Mining \& Knowledge Management Process (IJDKP) vol.3, no.5, September 2013.

[10] Edin Osmanbegović , Mirza Suljić . DATA MINING APPROACH FOR PREDICTING STUDENT PERFORMANCE. Economic Review - Journal of Economics and Business, vol. X, Issue 1, May 2012.

[11] U . K. Pandey, and S. Pal, "Data Mining: A prediction of performer or underperformer using classification", (IJCSIT) International Journal of Computer Science and Information Technology, vol. 2(2), pp.686-690, ISSN:0975-9646, 2011.

[12] Z. N. Khan, "Scholastic achievement of higher secondary students in science stream", Journal of Social Sciences, vol. 1, no. 2, pp. 84-87, 2005.

[13] Brijesh Kumar Baradwaj, Saurabh Pal. Mining Educational Data to Analyze Studentse Performance, (IJACSA) International Journal of Advanced Computer Science and Applications, vol. 2, no. 6, 2011. 